\title{
Dynamical characteristics of ice supersaturated regions
}

\author{
K. Gierens and S. Brinkop \\ Deutsches Zentrum für Luft- und Raumfahrt, Institut für Physik der Atmosphäre, Oberpfaffenhofen, Germany \\ Correspondence to: K. Gierens (klaus.gierens@dlr.de)
}

Received: 25 July 2012 - Published in Atmos. Chem. Phys. Discuss.: 9 August 2012

Revised: 31 October 2012 - Accepted: 6 December 2012 - Published: 17 December 2012

\begin{abstract}
The typical distributions of dynamical fields within ice supersaturated regions are investigated. The dynamical fields divergence, relative vorticity, and vertical velocity are analysed statistically in two ways, namely using the unconditioned data and data conditioned on the presence of ice supersaturation. Two geographical regions are considered, namely Europe $(250 \mathrm{hPa}$ level) and the tropical belt from $30^{\circ} \mathrm{S}$ to $30^{\circ} \mathrm{N}$ on two pressure levels (200 and $150 \mathrm{hPa}$ ). The study is based on forecast data from the European Centre for Medium-Range Weather Forecasts for four months covering the four seasons, June, September, December 2011 and March 2012. We find that histograms (frequency distributions) and low order moments of the dynamical fields differ substantially and statistically significantly inside and outside of ice supersaturated regions. As expected, upward and divergent flow favours ice supersaturation. But we find also that ice supersaturation is mostly located in anticyclonic flow. The latter result is probably due to the structure of warm/moist and cold/dry air streams in synoptic disturbances in mid-latitudes, but probably merely coincidental in the tropical belt.
\end{abstract}

\section{Introduction}

Ice supersaturated regions (ISSRs, for a recent review see Gierens et al., 2012) are peculiar regions in the upper troposphere with relative humidity with respect to ice (RHi) exceeding saturation. There is a tacit understanding that the temperature of ISSRs should be lower than the supercooling limit of liquid water (i.e. $T \leq-38^{\circ} \mathrm{C}$ ) in order not to count mixed phase clouds as ISSRs (water saturation within a mixed phase cloud implies ice supersaturation). In-situ formation of cirrus clouds is possible (i.e. formation by slow uplift) and aircraft condensation trails can persist for hours only in ISSRs. As in-situ cirrus formation usually requires quite high values of ice supersaturation, ISSRs are often cloud free. Ice supersaturation also occurs within cirrus clouds, but naturally there is a tendency to approach saturation due to growing ice crystals.

ISSRs are special in other respects as well. For instance, we find on average lower temperatures and higher specific humidities within ISSRs than in the remaining parts of the upper troposphere (Gierens et al., 1999; Spichtinger et al., 2003b; Spichtinger, 2004). This might point to formation of ice supersaturation by lifting of air masses or might alternatively mean that ISSRs form predominantly when and where it is relatively cold and moist. Further properties of ISSRs have been investigated during the past two decades, for instance the statistical distribution of the degree of supersaturation (an exponential distribution, Gierens et al., 1999; Spichtinger et al., 2002), their relation to sub-visible cirrus (Gierens et al., 2000), the geometrical properties horizontal and vertical extension (Gierens and Spichtinger, 2000; Spichtinger et al., 2003a), and satellite data derived climatologies (Spichtinger et al., 2003b; Gierens et al., 2004; Gettelman et al., 2006). However, characteristic differences between dynamical fields inside and outside of ISSRs have never been analysed before.

From case studies (Spichtinger et al., 2005b,a), weather analyses (Irvine et al., 2012) and from satellite data analyses including cirrus and contrail occurrence (Kästner et al., 1999; Wylie, 2002; Spichtinger et al., 2003b) we know that ISSRs occur preferentially in relation to certain synoptic weather patterns, e.g. the storm tracks and jet streams. Wylie (2002) mentions cumulonimbus convection, baroclinic fronts and lows and orographic lifting as cirrus generation mechanisms that are of interest here. Kästner et al. (1999) found the majority of automatically detected persistent contrails in divergent flow patterns in the upper troposphere in slowly rising 
warm (ahead a surface warm front) or locally turbulent cold air masses (ahead a surface cold front near a band of strong wind). These results indicate that also ISSRs occur related to certain dynamical features and this expectation will be tested in the current paper.

For such a study, we need fields of relative humidity and dynamics for large geographic regions. Such data are currently only available from Numerical Weather Prediction (NWP) models. A study like this would have been impossible ten years ago when ice supersaturation was generally not allowed in weather forecast models as all excess vapour was immediately turned into precipitation. Fortunately, this situation has changed and ice supersaturation is nowadays represented in at least some weather forecast models. For instance, the European Centre for Medium-Range Weather Forecasts (ECMWF) implemented a new scheme in its Integrated Forecast System (IFS) in 2006 (Tompkins et al., 2007). Here we use operational forecast data from the deterministic system. In this way, our study may as well be understood as an evaluation of the representation of ice supersaturation in the IFS. The retrieval procedure and data handling are described in the Sect. 2. Results in the form of frequency histograms and low order moments are presented and discussed in Sects. 3 and 4. Finally, conclusions are drawn in Sect. 5.

\section{Data treatment}

The current investigation is based on operational forecast data from the ECMWF IFS. We use the forecast with a lead time of $24 \mathrm{~h}$ for each day in June, September and December 2011 and March 2012, thus covering four seasons with one month each. An update to the IFS (cycle 37r3) on 15 November 2011 with modifications in the treatment of supersaturation does not affect the current study, since this update only affects mixed-phase clouds but does not affect ice supersaturation in the upper troposphere (Richard Forbes, private communication, 2012). We consider two regions, Europe $\left(27^{\circ} \mathrm{W}\right.$ to $45^{\circ} \mathrm{E}, 33^{\circ} \mathrm{N}$ to $\left.73.5^{\circ} \mathrm{N}\right)$ on the $250 \mathrm{hPa}$ level, and the tropical belt $\left(30^{\circ} \mathrm{S}\right.$ to $\left.30^{\circ} \mathrm{N}\right)$ on 150 and $200 \mathrm{hPa}$. Tropical data are only analysed for March 2012 because seasonal variations should be weak in the tropics. Europe is considered here a typical mid-latitude region, and the tropical levels are selected because ISSRs are known to be frequent there (Spichtinger et al., 2003b). The spectral T1279 data are interpolated to a spatial resolution of $0.25^{\circ} \times 0.25^{\circ}$. For Europe we use forecasts initialised at 00:00 and 12:00 UTC (i.e. 60 or 62 data sets per month) while for the tropics we only use the forecast initialised at 00:00 UTC (31 data sets). The monthly mean fractional coverage (on the selected pressure level) of ISSRs is in the considered months $9.3(12.5,10.8$, 8.8) \% for Europe (with a total range from $2.8 \%$ to $25.1 \%$ ), consistent with recent results from Irvine et al. (2012) who note a low bias of the occurrence frequencies of ISSRs in the ECMWF re-analyses (ERA-interim). The corresponding val- ues are $10.3 \%$ (8.9\% to $11.7 \%)$ and $9.5 \%$ (7.7\% to $11.7 \%)$ in the tropical belt on 200 and $150 \mathrm{hPa}$, respectively. These numbers are low biased as well, as comparisons with various data sources can show, for instance satellite data (Spichtinger et al., 2003b; Lamquin et al., 2012) and MOZAIC data (Measurement of Ozone and Water Vapor by Airbus In-Service Aircraft, Luo et al., 2007, 2008). Low biases of ISSR occurrence, however, are not detrimental to our study as long as they are modelled at the right locations and in the right proportions. This quality is usually conceded to the IFS model (Luo et al., 2008; Irvine et al., 2012).

We analyse the following dynamical fields: horizontal divergence $(\delta=\partial u / \partial x+\partial v / \partial y)$, relative vorticity ( $\zeta=$ $\partial v / \partial x-\partial u / \partial y$ ), and the vertical velocity $\omega$ (in pressure coordinates, units $\mathrm{Pa} \mathrm{s}^{-1}$, that is, negative values signify upward motion). These fields and the relative humidity field have been retrieved on standard pressure levels. Other dynamical fields like potential vorticity cannot easily be retrieved from that archive where relative humidity is stored and are therefore not considered. Low temperatures on $250 \mathrm{hPa}$ and higher up imply according to the implementation in the IFS that the relative humidity is relative to ice and values exceeding $100 \%$ signify ice supersaturation (that is, an ISSR). We take the relative humidity field without considering the possible existence of clouds in the same grid box (a fully cloud covered grid box has no supersaturation by implementation because the model time step is considered long enough that all initial supersaturation within a cloud can be consumed by crystal growth, see Tompkins et al., 2007).

We compute frequency distributions (histograms) of the dynamical fields in the selected regions from all data and restricted to ISSRs for the complete month. The histograms are normalised to mode values (maxima) of unity and are shown up to \pm 3 standard deviations of the whole respective data set $\left(\sigma_{\text {all }}\right)$. Additionally we compute first the four lowest moments for each single forecast (indexed with $i$ ). These low moments are mean $\mu_{i}$, standard deviation $\sigma_{i}$, skewness $S_{i}$ and kurtosis $\kappa_{i}$. For a set of data $\xi_{n i}$ given at $n=1 \ldots N$ grid points they are defined as:

$$
\begin{aligned}
\mu_{i} & =\frac{1}{N} \sum_{n=1}^{N} \xi_{n i}, \\
\sigma_{i} & =\left[\frac{1}{N-1} \sum_{n=1}^{N}\left(\xi_{n i}-\mu_{i}\right)^{2}\right]^{1 / 2}, \\
S_{i} & =\frac{1}{N} \sum_{n=1}^{N}\left(\frac{\xi_{n i}-\mu_{i}}{\sigma_{i}}\right)^{3} \\
\kappa_{i} & =\frac{1}{N} \sum_{n=1}^{N}\left(\frac{\xi_{n i}-\mu_{i}}{\sigma_{i}}\right)^{4}-3 .
\end{aligned}
$$

Then these four quantities are averaged over all 60, 62 or 31 forecasts (giving $\mu, \sigma, S$, and $\kappa$ ) and the corresponding standard deviations are computed in order to see how variable $\mu_{i}, \sigma_{i}, S_{i}$, and $\kappa_{i}$ are in the course of the month and 
Table 1. Statistical parameters mean, standard deviation, skewness and kurtosis, of dynamical fields within ISSRs and generally in three geographic regions on appropriate pressure levels. Mean values and standard deviations of divergence and vorticity (units $\mathrm{m}^{2} \mathrm{~s}^{-2}$ ) have been scaled by $10^{6}$, while skewness and kurtosis are dimensionless. For the meaning of the parameters and their standard deviations see the text. Values that are at least three times larger than their standard deviation are printed in boldface.

\begin{tabular}{|c|c|c|c|c|c|c|c|c|c|c|}
\hline & \multicolumn{2}{|c|}{ Europe $(250 \mathrm{hPa})$} & \multicolumn{2}{|c|}{ Tropics SH $(150 \mathrm{hPa})$} & \multicolumn{2}{|c|}{ Tropics NH $(150 \mathrm{hPa})$} & \multicolumn{2}{|c|}{ Tropics SH $(200 \mathrm{hPa})$} & \multicolumn{2}{|c|}{ Tropics NH $(200 \mathrm{hPa})$} \\
\hline & all & ISSR & all & ISSR & all & ISSR & all & ISSR & all & ISSR \\
\hline \multicolumn{11}{|c|}{ divergence } \\
\hline mean & $-0.05 \pm 0.50$ & $15.3 \pm 4.7$ & $0.78 \pm 0.38$ & $23 \pm 1.8$ & $-0.83 \pm 0.37$ & $16 \pm 3.0$ & $0.82 \pm 0.32$ & $12 \pm 1.7$ & $-0.95 \pm 0.30$ & $12 \pm 2.5$ \\
\hline std. dev. & $\mathbf{3 0} \pm \mathbf{3}$ & $29 \pm 5$ & $32 \pm 1$ & $41 \pm 2$ & $26 \pm 1$ & $32 \pm 4$ & $32 \pm 1$ & $49 \pm 2$ & $26 \pm 1$ & $42 \pm 2$ \\
\hline skewness & $-0.05 \pm 0.27$ & $1.0 \pm 0.6$ & $0.7 \pm 0.2$ & $1.1 \pm 0.3$ & $0.4 \pm 0.4$ & $1.9 \pm 0.9$ & $0.9 \pm 0.2$ & $0.7 \pm 0.3$ & $0.6 \pm 0.2$ & $1.1 \pm 0.5$ \\
\hline kurtosis & $7.8 \pm 4.7$ & $4.0 \pm 3.3$ & $9.0 \pm 6.2$ & $5.8 \pm 5.2$ & $14 \pm 11$ & $14 \pm 21$ & $10.0 \pm 4.3$ & $5.1 \pm 3.2$ & $10.6 \pm 3.1$ & $7.2 \pm 4.0$ \\
\hline \multicolumn{11}{|c|}{ rel. vorticity } \\
\hline mean & $2.6 \pm 3.9$ & $-58 \pm 13$ & $3.8 \pm 1.3$ & $13 \pm 2.6$ & $-7.5 \pm 2.3$ & $-13 \pm 3.7$ & $4.7 \pm 1.1$ & $15 \pm 2.5$ & $-8.4 \pm 2.0$ & $-15 \pm 4.3$ \\
\hline std. dev. & $64 \pm 5$ & $38 \pm 9$ & $\mathbf{3 8} \pm \mathbf{1}$ & $43 \pm 3$ & $\mathbf{3 1} \pm \mathbf{2}$ & $\mathbf{3 3} \pm \mathbf{3}$ & $45 \pm 2$ & $49 \pm 3$ & $40 \pm 2$ & $44 \pm 4$ \\
\hline skewness & $0.39 \pm 0.22$ & $0.81 \pm 0.36$ & $-0.33 \pm 0.26$ & $-0.25 \pm 0.61$ & $0.57 \pm 0.32$ & $0.31 \pm 0.32$ & $-0.68 \pm 0.35$ & $-0.23 \pm 0.72$ & $0.87 \pm 0.26$ & $0.24 \pm 0.30$ \\
\hline kurtosis & $0.12 \pm 0.76$ & $1.3 \pm 1.2$ & $4.0 \pm 2.9$ & $5.6 \pm 7.9$ & $3.1 \pm 1.2$ & $4.2 \pm 1.5$ & $5.2 \pm 4.4$ & $6.5 \pm 11.3$ & $3.2 \pm 1.2$ & $2.9 \pm 1.1$ \\
\hline \multicolumn{11}{|c|}{ vertical velocity } \\
\hline mean & $0.001 \pm 0.005$ & $-0.047 \pm 0.024$ & $-0.003 \pm 0.002$ & $-0.075 \pm 0.011$ & $-0.002 \pm 0.001$ & $-0.045 \pm 0.012$ & $-0.008 \pm 0.003$ & $-0.15 \pm 0.01$ & $0.007 \pm 0.002$ & $-0.097 \pm 0.016$ \\
\hline std. dev. & $0.12 \pm 0.02$ & $0.13 \pm 0.04$ & $0.09 \pm 0.01$ & $0.13 \pm 0.02$ & $0.08 \pm 0.01$ & $0.10 \pm 0.02$ & $0.13 \pm 0.01$ & $0.20 \pm 0.02$ & $0.11 \pm 0.01$ & $0.16 \pm 0.03$ \\
\hline skewness & $-0.7 \pm 0.7$ & $-1.1 \pm 0.9$ & $-2.3 \pm 1.0$ & $-2.9 \pm 1.1$ & $-1.4 \pm 1.1$ & $-3.0 \pm 1.5$ & $-2.9 \pm 0.9$ & $-2.8 \pm 0.9$ & $-1.9 \pm 1.9$ & $-3.4 \pm 2.5$ \\
\hline kurtosis & $22 \pm 14$ & $10 \pm 10$ & $33 \pm 30$ & $20 \pm 22$ & $56 \pm 46$ & $30 \pm 48$ & $38 \pm 29$ & $23 \pm 21$ & $75 \pm 89$ & $45 \pm 86$ \\
\hline
\end{tabular}

whether values are statistically different from zero. For clarity, we give an example (let $I$ be the number of forecasts analysed):

$$
\begin{aligned}
\sigma & =\frac{1}{I} \sum_{i=1}^{I} \sigma_{i} \\
\sigma_{\sigma} & =\left[\frac{1}{I-1} \sum_{i=1}^{I}\left(\sigma_{i}-\sigma\right)^{2}\right]^{1 / 2} .
\end{aligned}
$$

Thus, $\sigma$ is the mean of the daily (or twice daily) standard deviations $\sigma_{i}$, and $\sigma_{\sigma}$ is the standard deviation of the distribution of the individual $\sigma_{i}$, which can be exploited to analyse statistical significance. Analogous procedures are applied for mean, skewness and kurtosis. These quantities are presented in Table 1 . Note that the mean $\mu$ is indeed the monthly mean (as taken at once over all $i$ and $n$ ) while this is not so for the other moments (because their determination involves nonlinear operations), for instance:

$\sigma_{\mathrm{all}}=\left[\frac{1}{I N-1} \sum_{i=1}^{I} \sum_{n=1}^{N}\left(\xi_{n i}-\mu\right)^{2}\right]^{1 / 2}$,

and this is slightly different from $\sigma$ above.

\section{Results}

In this section we describe in some detail results for March 2012 because for this month we have analysed both Europe and the tropics. The results for the other three months are qualitatively very similar to the March results and are presented in the Supplement to this paper.

The frequency distributions of divergence, relative vorticity, and vertical wind speed are plotted as histograms in Figs. 1 to 3. As mentioned, the overall standard deviations $\sigma_{\text {all }}$ of the respective field (all times and grid points) have been used to define the bins and to normalise the data by division of all single values with $\sigma_{\text {all }}$. Note that the same $\sigma_{\text {all }}$ is used for both the data of a region as a whole (unconditioned distribution) and the ISSR-restricted data (ISSR-conditioned distribution). Large differences between these unconditioned and ISSR-conditioned distributions are immediately evident for all regions (all months, see Supplement) and all quantities considered.

For instance, the ISSR-conditioned distribution of divergence is shifted to the right (positive values) of the respective unconditioned distribution in all regions and in the tropics on $200 \mathrm{hPa}$ it is also much broader. The divergence distributions seem to be close to Gaussian. There are no substantial differences between corresponding distributions from southern hemispheric $(\mathrm{SH})$ and northern hemispheric $(\mathrm{NH})$ tropical distributions.

The tropical vorticity distributions look slightly skewed, with different sense in the two hemispheres, but most skewness values are insignificant (see Table 1). The vorticity mean values of the ISSR-conditioned distributions are roughly 2 to 4 times larger than averages of the corresponding unconditioned data. However, since the vorticity standard deviations are about 10 times larger than the mean values of the unconditioned data, the differences in the mean of the unconditioned and ISSR-conditioned data are not spectacularly visible in the histograms. The two vorticity distributions differ most for the mid-latitude region of Europe. Both are clearly non-Gaussian and their shapes differ strongly. While we have a very broad unconditioned vorticity distribution with a mean close to zero, the ISSR-conditioned vorticity distribution is narrower, but with a strongly negative mean value and hardly any positive values. Seemingly, most European ISSRs in the analysed dataset were located in anti-cyclonic flows. This 

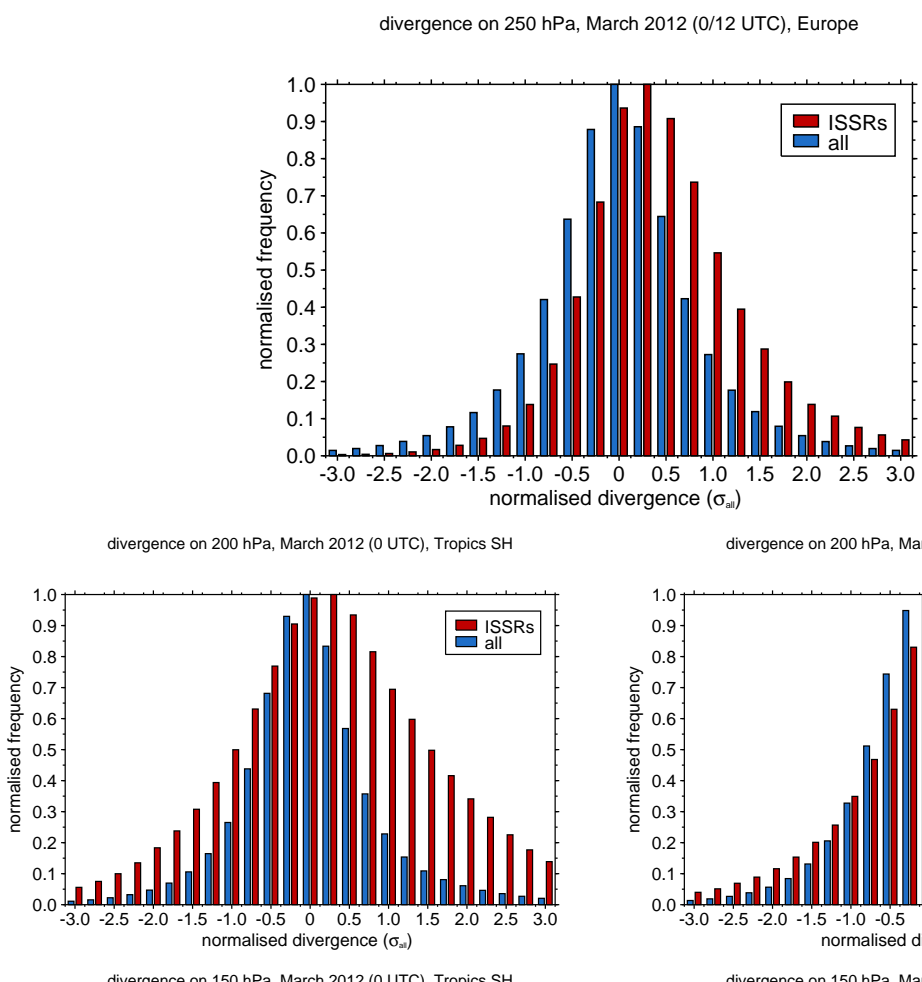

divergence on $200 \mathrm{hPa}$, March 2012 (0 UTC), Tropics NH
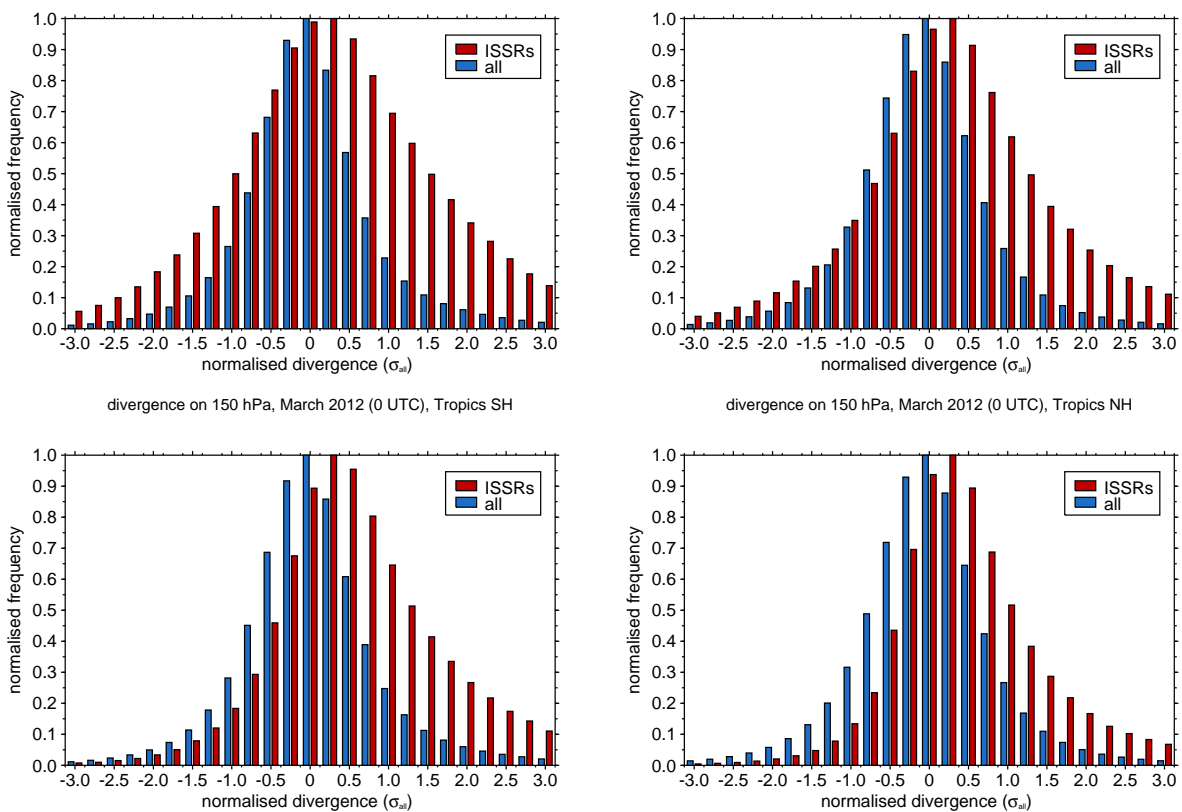

divergence on $150 \mathrm{hPa}$ March 2012 (O UTC), Tropics NH

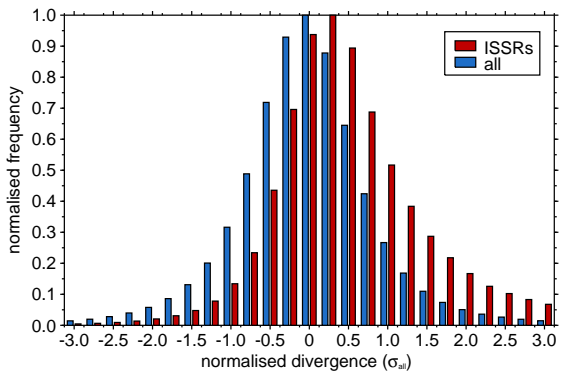

Fig. 1. Histograms of divergence for various regions: Europe on the $250 \mathrm{hPa}$ level (top row), and the tropics divided in Northern (NH, right) and Southern Hemisphere (SH, left) on 200 (middle row) and $150 \mathrm{hPa}$ levels (bottom row). The blue bars refer to all grid boxes in the respective region while the red ones refer to ISSRs only. The data are normalised with the standard deviation of the complete set of all forecast times and all grid boxes $\left(\sigma_{\text {all }}\right)$. The relative frequencies of occurrence are normalised such that the maximum value is unity. The bin width is $\sigma_{\text {all }} / 4$ and the bins are marked with the small ticks on the x-axes.

result holds as well for the other considered months (Supplement).

The mean vertical velocities of the unconditioned distributions are close to zero, but those within ISSRs are clearly negative and generally their magnitude is an order of magnitude larger than the former. We find slightly negatively skewed distributions for the unconstrained data, but distinctively negatively skewed distributions within ISSRs. The tropical histograms are mutually similar and they are also similar to the histogram of the European data. As one might expect, uplifting prevails in ISSRs.

Thus a major result of this study is: ice supersaturation is not equally common to all flow patterns and it is preferentially related to certain flow types, in particular upward motion (as expected), anti-cyclonic flow in northern midlatitudes, and divergent flow in the mid-latitudes and the tropics. However, except for the vorticity over Europe, all distributions overlap to a large degree, which firstly reminds us that questions of statistical significance have to be considered (see below) and secondly shows that there are hardly any flow patterns that are forbidden for the flow in ISSRs. Instead, the differences mean only that the relative frequency of certain patterns is different within and outside of ISSRs.

Let us now analyse the entries in Table 1 with the following understanding: we consider a value $(\mu, \sigma, S, \kappa)$ significantly different from zero (printed bold in the table) if its absolute value exceeds at least three times its day-to-day variability (that is, $\sigma_{\mu}, \sigma_{\sigma}, \sigma_{S}, \sigma_{\kappa}$ ). The entries in the table are in 
vorticity on $250 \mathrm{hPa}$, March 2012 (0/12 UTC), Europe

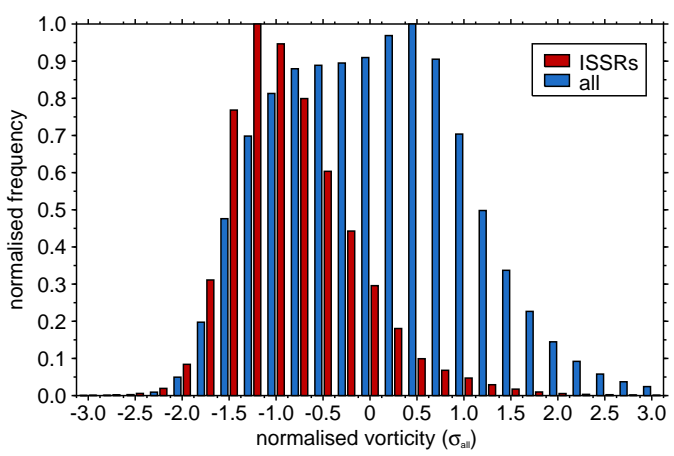

vorticity on $200 \mathrm{hPa}$, March 2012 (0 UTC), Tropics SH

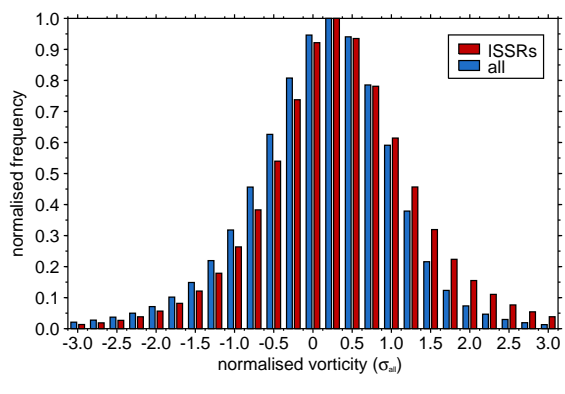

vorticity on $150 \mathrm{hPa}$, March 2012 (0 UTC), Tropics SH

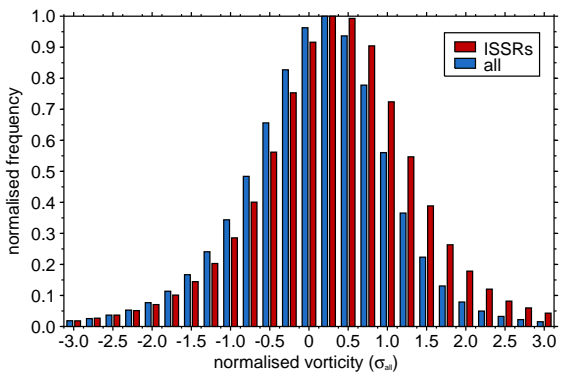

Fig. 2. As Fig. 1, but for relative vorticity.

the form $S \pm \sigma_{S}$, for instance. The 3- $\sigma$ criterion is fairly strict as less than one percent of the data of a Gaussian distribution lie outside the $\pm 3 \sigma$ boundaries. Insignificant values imply that the daily variability of that quantity is relatively strong compared with its monthly average and sign changes occur frequently.

We start with the divergence. The unconditioned ("all") mean divergences are almost all nearly zero and their deviations from zero are insignificant, given the variations from day to day, except for the $\mathrm{NH}$ tropics at $200 \mathrm{hPa}$. Contrary to this, ISSRs are regions of positive divergence, and the deviations from zero are significant in all considered regions. All mean divergences in ISSRs are at least an order of magnitude larger than the absolute value of the only significant mean divergence in the unconditioned data. The mean standard deviations $\sigma$ are roughly twice as large as the mean divergences within ISSRs and the daily $\sigma_{i}$ do not vary much during the month, i.e. $\sigma_{\sigma}$ is generally much smaller than $\sigma$ (about an order of magnitude). The divergence distributions within tropical ISSRs are considerably wider than the unconditioned divergence distributions in the same regions. There are significant values of skewness both in the unconditioned and ISSR-conditioned data in the SH tropics on $150 \mathrm{hPa}$, and another one in the unconditioned data of the $\mathrm{NH}$ tropics at $200 \mathrm{hPa}$. All other skewness and all kurtosis values are insignificant. This is consistent with our earlier impression that the histograms of divergence look Gaussian.

In almost all regions we find mean vorticities that are significantly different from zero, except the unconditioned data of Europe and the SH tropics at $150 \mathrm{hPa}$. The latter average, however, is close to significant on the 3- $\sigma$ level. The (absolute) mean vorticities of the ISSR-conditioned distributions are much larger than the unconditioned mean values of the same region and their significance are generally on a higher 
vert. velocity on $250 \mathrm{hPa}$, March 2012 (0/12 UTC), Europe

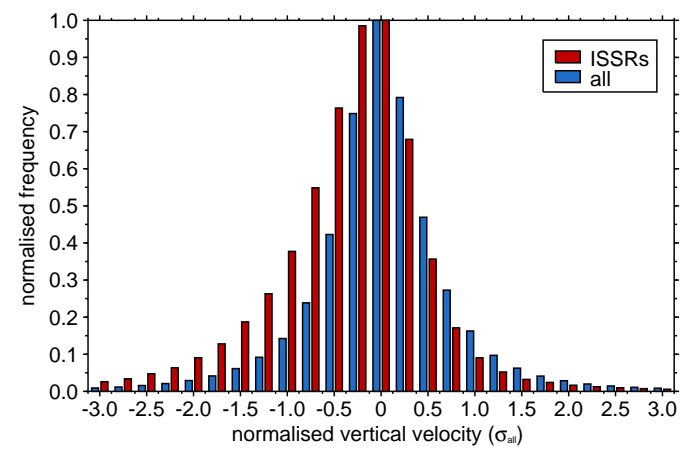

vert. velocity on $200 \mathrm{hPa}$, March 2012 (0 UTC), Tropics SH

vert. velocity on $200 \mathrm{hPa}$, March 2012 (0 UTC), Tropics NH
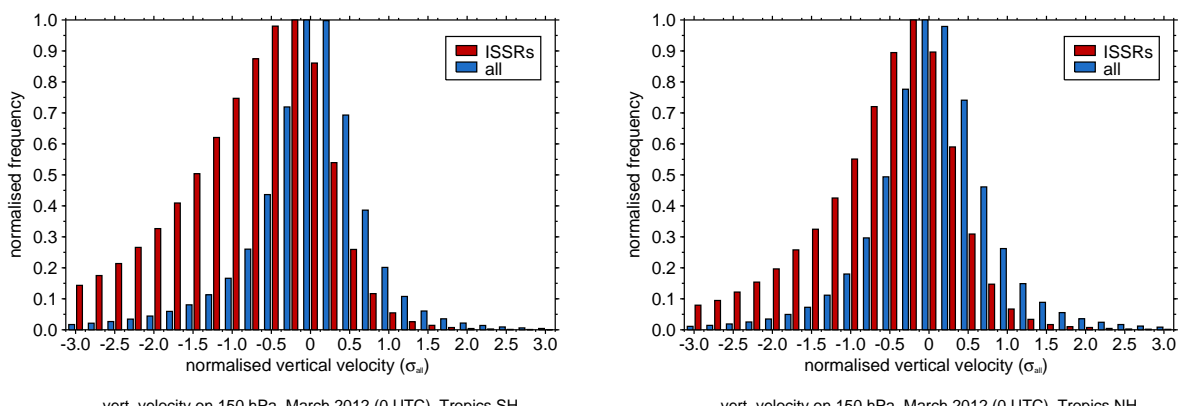

vert. velocity on $150 \mathrm{hPa}$, March 2012 (0 UTC), Tropics NH
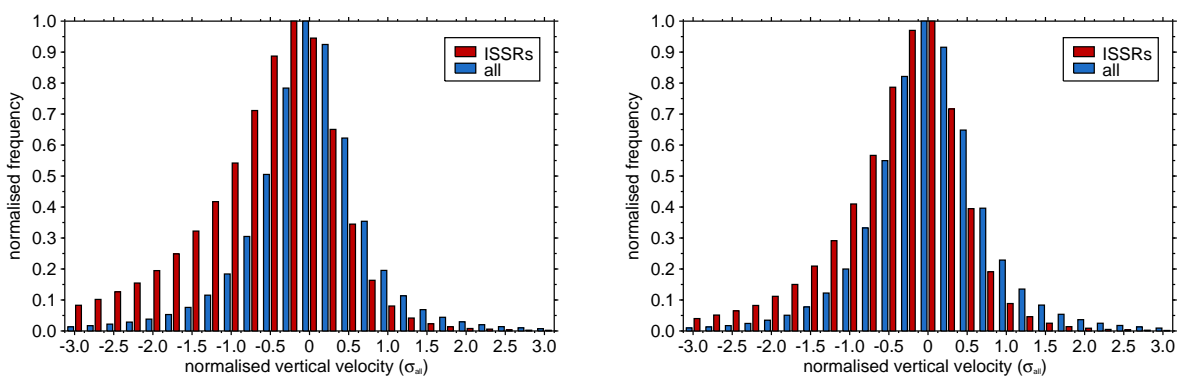

Fig. 3. As Fig. 1, but for vertical velocity, $\omega=\mathrm{d} p / \mathrm{d} t$. Negative values mean upward motion.

than 3- $\sigma$ level. The largest absolute vorticity mean value is found in the European ISSRs, which implies their preferential location in strongly anti-cyclonic flow. This strong mean value is even larger than the corresponding monthly mean standard deviation, that is, a positive mean vorticity in European ISSRs was hardly present during March 2012 and the other months (see the Table and Fig. 2 in the Supplement). In the tropics the standard deviations are much larger than the mean values, so that over the course of the month the mean daily vorticity sometimes changes sign. Skewness and kurtosis are insignificant with one exception. Surprisingly, this exception is not the unconditioned European vorticity distribution with its fairly strange shape, but the "all" distribution in the $\mathrm{NH}$ tropics at $200 \mathrm{hPa}$, which is slightly positively skewed.

Considering the vertical velocities we expect to find on average uplift in the ISSRs because uplift implies cooling and cooling implies rising relative humidity. Indeed we find nega- tive mean values of $\omega$ in the ISSR data which are very signif-

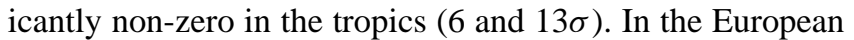
data the 3- $\sigma$ significance level is reached in June and December 2011, it is just missed in September 2011 (see Supplement Table) and relatively clearly missed in March 2012. The unconditioned data have a significant mean value only in the $\mathrm{NH}$ tropics at $200 \mathrm{hPa}$, all other monthly mean values are insignificantly different from zero. The daily fluctuation of the mean values is quite large, typically twice the absolute value of the mean in the ISSRs, but one to two orders of magnitude larger than the averages in the whole regions, probably reflecting the variation caused by the migrating synoptic systems over Europe and the cumulonimbus convection over the tropics. The skewness values are mostly insignificant, except for the SH tropics at $200 \mathrm{hPa}$ for both the unconditioned and ISSR-conditioned data sets, where the data are negatively skewed (signifying preference of upward motion). Admittedly the histograms appear to show negative skewness 

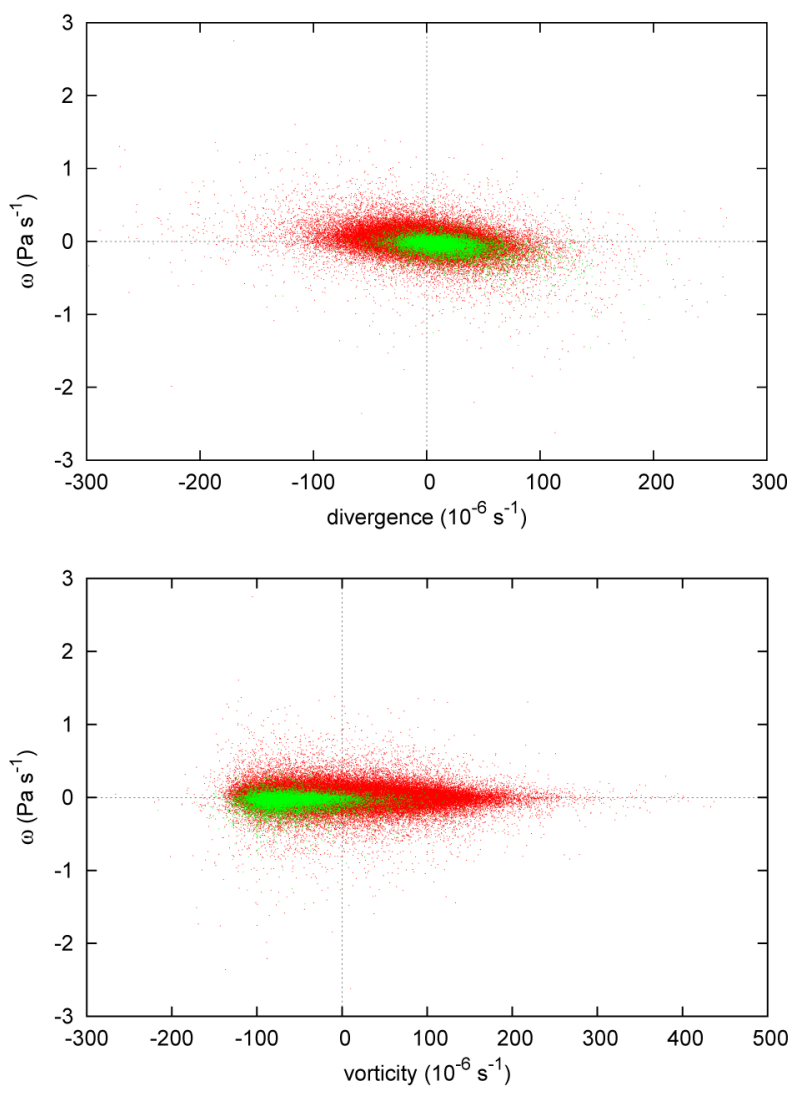

Fig. 4. Scatter plot of vertical velocity, $\omega=\mathrm{d} p / \mathrm{d} t$, and divergence (top panel) or vorticity (bottom panel) for a reduced sample of the Europe data. Red dots refer to all humidity values, green dots to ice superaturated areas only.

in all data sets, but the strict 3- $\sigma$ criterion lets skewness be significant in only the two mentioned cases. The kurtosis is generally not significantly different from zero.

\section{Discussion and interpretation}

We have seen that ISSRs are often located in uplifting, divergent and anti-cyclonic flow patterns. We expect that uplifting prevails in ISSRs because the excess moisture must eventually come from lower layers and because uplifting implies cooling and thus increase of relative humidity (e.g. Gierens et al., 1999; Spichtinger et al., 2003b). Spichtinger et al. (2003a) found from a radiosonde based study that ISSRs occur predominantly in a roughly $200 \mathrm{hPa}$ thick layer beneath the tropopause in moist air masses that are mostly colder than $-40^{\circ} \mathrm{C}$. Therefore uplifting of air masses simultaneously has the tendency to lead to ice supersaturation and to divergence because of the vertical lid of the tropopause, such that ISSRs and positive divergence could both be consequences of uplift close to the tropopause. If this is indeed the case there should be some correlation between the fields. Surprisingly, the correlation between $\omega$ and $\delta$ is weak. For the European dataset
(March 2012) the correlation between $\omega$ and $\delta$ is -0.30 for all data, and -0.35 for the ISSRs, cf. Fig. 4 (top panel). The correlations for the other months are similar with a maximum of -0.5 for December 2011; actually their absolute values must be even smaller, because both fields are autocorrelated themselves which we have not regarded in the determination of the correlation factor. In the IFS the equation for $\omega$ has two contributions, one related to the vertical profile of $\delta$ and one related to the angle between isobars and horizontal planes (a geometric correction). Since $\delta$ enters our analysis only on a single level instead of the whole column and because of the additional geometric correction the surprise that $\omega$ and $\delta$ are only weakly correlated is mitigated. The correlation between divergence and $\omega$ is even weaker in the tropics (we only tested the NH $200 \mathrm{hPa}$ data), and there is no correlation between the vertical wind and vorticity (Fig. 4, bottom panel). Thus the conditions uplift, positive divergence and anti-cyclonic flow are indeed independent of each other, both generally but as well within ice supersaturated regions.

Some ISSR events are untypically related to a downdraught, to convergence or to cyclonic motion. These are probably relatively old ISSRs that either have been advected to such locations or regions where the conditions since formation have reversed.

The scatter plots of Fig. 4 reveal another interesting feature, namely the green dots referring to ISSR data points are completely surrounded by red points, which refer to all data. Thus, ISSRs are not characterised to have the extreme values in the distributions. All parameter combinations of $\delta, \zeta$, $\omega$ that have been found for ISSRs can also be found in other regions. Therefore, in the sense of regression analysis, these dynamical fields alone are insufficient for a prediction of ice supersaturation. The thermodynamic properties of an airmass (moisture, temperature) are at least as important for such a purpose.

Results from independent data sources using cirrus and contrail appearance as proxies might corroborate or contradict ours. Thus we can look how cirrus clouds and persistent contrails are situated in weather patterns. Wylie (2002) reviews older analyses of cirrus occurrence in relation to the jet stream and Rossby waves. He shows dense and opaque cirrus to occur close to the ridges of Rossby waves where the flow is anti-cyclonic. On the contrary, the sky is clear of cirrus in the cyclonic trough part of the Rossby wave. A case study of a long-lasting ISSR over Europe (Spichtinger et al., 2005b) shows the ISSR in anti-cyclonic flow along a high pressure ridge as well. Moist air ascends on the western side of the ridge in a warm conveyor belt and descends on the eastern side. Similar mechanisms to promote ISSR formation (slantwise ascent of warm air in storms growing on the jet stream) have been found by Irvine et al. (2012) in an analysis of the relation of ISSRs to different weather patterns. In situations where a pronounced ridge is located over the Atlantic they find ISSRs in the anti-cyclonic flow. Warm conveyor belts are dynamical features which Kästner et al. (1999) identify as 
appropriate for detection of persistent contrails. These results corroborate our finding that in the mid-latitudes ISSRs are predominantly found in anti-cyclonic flow. However, Kästner et al. (1999) detect almost as many contrails in cyclonic as in anti-cyclonic flow which is in contradiction to our results and those referred to by Wylie (2002). It might be that the contrails that are found in cyclonic flow are simply advected from their formation regions where the flow is anti-cyclonic.

Immler et al. (2008) find from very sensitive Lidar observations (that could detect cirrus down to an optical thickness of around $10^{-4}$ ) at Lindenberg (NE Germany) obtained during the July/August 2003 heat wave that cirrus are ubiquitous in high pressure systems and that ISSRs occur very often in anti-cyclonic flow. Yet cirrus cover can be very high also in low pressure dominated (cyclonic) situations, namely when it is related to the warm conveyer belt, which transports moist air from the lower troposphere upward and which is divergent at least at its upper end; cirrus cover is very low after the passage of the cold front because the upper tropospheric air in this sector has low absolute humidity and does not get into contact with the moister tropospheric regions during the synoptic development. These results show again that the dynamical fields alone are insufficient to predict ice supersaturation or cirrus clouds. The recent trajectory of an airmass is equally important (i.e. had the airmass contact to moist regions or not).

In the tropical UT in the upper branch of the Hadley cell ISSRs are mostly located where divergence and uplift speed are much larger than in the large-scale mean. Such regions are likely related to the outflow of deep convection which transports the necessary moisture into the UT from the moist lower troposphere. In the ISSRs we find that the mean divergence increases from 200 to $150 \mathrm{hPa}$ while the mean uplift speed decreases with altitude, which indicates that a vertical flow is decelerated and eventually turned into a horizontal outflow. These results are consistent with results from analyses of moisture flux divergence obtained from MOZAIC measurements in the tropics (Luo et al., 2007). However, again strong uplift and large divergence alone are not sufficient to imply ice supersaturation; we find such dynamical conditions also in air with low relative humidity. Figure 5 shows where in the tropical belt higher than typical (defined as $\delta>16 \times 10^{-6} \mathrm{~s}^{-1}$ ) divergence occurs frequently. These regions along the ITCZ are more or less congruent with regions where ISSRs are frequent, too. This map shows frequent ISSR occurrence where it was also found in satellite data, the Microwave Limb Sounder (Spichtinger et al., 2003b) and the Atmospheric Infrared Sounder (Gettelman et al., 2006; Lamquin et al., 2012).

A different picture is obtained by looking in the same way at the vorticity, see Fig. 6 . Although the mean vorticity is about twice as large in ISSRs than in the rest of the tropics, it seems that this is likely coincidental because the moisture originating close to the ITCZ with predominant easterly winds flows poleward into zones with predominant westerly

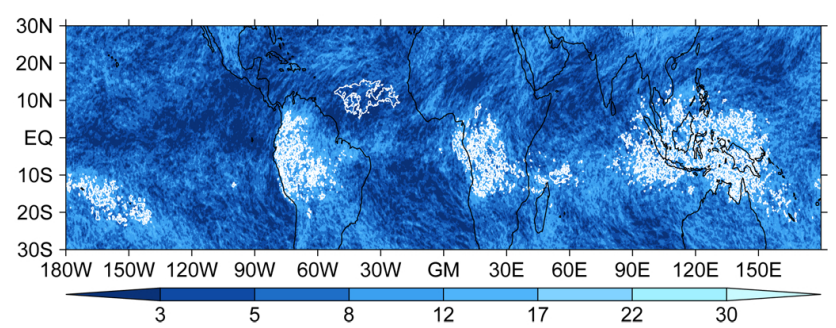

Fig. 5. Map of the tropical belt at $150 \mathrm{hPa}$ highlighting regions where higher than average divergence occurs often (brighter colours mean more events, see colourbar) and where ice supersaturation occurs at least on ten of the 31 March days (white contours). Higher than average divergence is defined here as $\delta>16 \times 10^{-6} \mathrm{~s}^{-1}$.

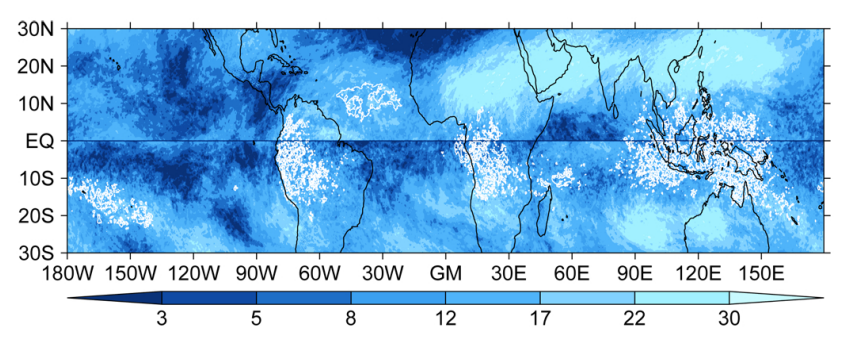

Fig. 6. Map of the tropical belt at $150 \mathrm{hPa}$ highlighting regions where higher than average vorticity occurs often (brighter colours mean more events, see colourbar) and where ice supersaturation occurs at least on ten of the 31 March days (white contours). Higher than average vorticity is defined here as $\zeta<-13 \times 10^{-6} \mathrm{~s}^{-1}$ in the $\mathrm{NH}$ and $\zeta>13 \times 10^{-6} \mathrm{~s}^{-1}$ in the $\mathrm{SH}$.

winds (Fig. 6 of Luo et al., 2007), which is anti-cyclonic rotation. In fact, ISSRs mostly appear in regions where more extreme vorticities appear relatively frequently. But while the highest occurrence frequencies of ISSRs occur close to the equator (ITCZ) on the $150 \mathrm{hPa}$ level, the highest occurrences of large vorticity values (here chosen as $\zeta<-13 \times 10^{-6} \mathrm{~s}^{-1}$ in the $\mathrm{NH}$ and $\zeta>13 \times 10^{-6} \mathrm{~s}^{-1}$ in the $\mathrm{SH}$ ) are located close to the subtropical subsidence zones where ISSRs usually do not occur. Ice supersaturation is also found in anticyclonic flow related to Rossby wave breaking on the subtropical tropopause (Waugh, 2005).

\section{Conclusions}

We have performed a statistical analysis of the dynamical fields divergence, relative vorticity, and vertical velocity within ISSRs and outside for two geographical regions, namely Europe $(250 \mathrm{hPa}$ level) and the tropical belt from $30^{\circ} \mathrm{S}$ to $30^{\circ} \mathrm{N}$ on two pressure levels (200 and $150 \mathrm{hPa}$ ). The study was based on $24 \mathrm{~h}$ forecast data from the ECMWF for March 2012 (with 00:00 UTC initialisation for the tropics and 00:00 and 12:00 UTC initialisation for Europe). For Europe we have additionally analysed June, September, and December 2011 (see the Supplement) in order to see whether 
there are seasonal variations; these turned out to be negligible. We find that histograms (frequency distributions) and low order moments mean, standard deviation, and partly skewness and kurtosis, of the dynamical fields differ substantially when the data are either unconditioned (i.e. all data are used) or conditioned on ice supersaturation (i.e. only data within ISSRs are used). The results can be summarised as follows:

- Upward wind and divergent airflow are favourable conditions for the formation of ice supersaturation. But ISSRs are not always present when these conditions are met. Ice supersaturation is rare in regions with downward or convergent flow.

- ISSRs in Northern mid-latitudes are mainly confined to regions with anti-cyclonic flow. This is probably explainable by the organisation of the warm/moist and cold/dry air streams in mid-latitude synoptic disturbances - a kind of causal relation. In the tropics anticyclonic flow prevails due to the flow situation with mainly easterly wind at the equator and westerly wind northward and southward. Although the mean vorticity in tropical ISSRs is stronger anti-cyclonic than the corresponding average vorticity of the whole $\mathrm{NH}$ or SH part of the tropical belt, the relation of the appearance of ISSRs and anti-cyclonic flow seems to be coincidental.

As all parameter combinations $(\delta, \zeta, \omega)$ that occur in ISSRs occur in subsaturated air as well, it is not possible to forecast ISSRs using dynamical fields alone. The history of an air parcel has to be known as well, i.e. whether it was or was not in contact with moisture sources recently (e.g. Nawrath, 2002). But there are parameter combinations where the occurrence of ISSRs is extremely unlikely, such that even weather models without a representation of ice supersaturation can give good hints of regions where ice supersaturation is not to be expected. Such forecasts could be valuable for climate optimised, contrail avoiding planning of flight routes.

Overall our results are somehow expected and pose no difficulties for interpretation. They may be understood as a contribution to model evaluation of the IFS and lend thus more credibility to the representation of ice supersaturation in this forecast model.

\section{Supplementary material related to this article is available online at: http://www.atmos-chem-phys.net/12/ 11933/2012/acp-12-11933-2012-supplement.zip.}

Acknowledgements. The data for this study have been obtained from the European Centre for Medium-Range Weather Forecasts in the framework of the Special Project SPDEISSR. SB was funded via the European Commission's FP7 project REACT4C. This work contributes to the COST Action ES0604 WaVaCS (Atmospheric
Water Vapour in the Climate System). We are grateful to Andreas Dörnbrack for reading and discussing an earlier version of the manuscript.

Edited by: G. Vaughan

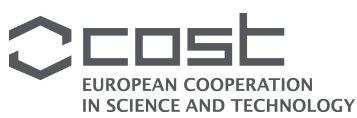

This publication is supported by COST - www.cost.eu

\section{References}

Gettelman, A., Fetzer, E., Eldering, A., and Irion, F.: The global distribution of supersaturation in the upper troposphere from the Atmospheric Infrared Sounder, J. Climate, 19, 6089-6103, 2006.

Gierens, K. and Spichtinger, P.: On the size distribution of icesupersaturated regions in the upper troposphere and lowermost stratosphere, Ann. Geophys., 18, 499-504, doi:10.1007/s00585000-0499-7, 2000.

Gierens, K., Schumann, U., Helten, M., Smit, H., and Marenco, A.: A distribution law for relative humidity in the upper troposphere and lower stratosphere derived from three years of MOZAIC measurements, Ann. Geophys., 17, 1218-1226, doi:10.1007/s00585-999-1218-7, 1999.

Gierens, K., Schumann, U., Helten, M., Smit, H., and Wang, P.: Ice-supersaturated regions and sub visible cirrus in the northern midlatitude upper troposphere, J. Geophys. Res., 105, 2274322754, 2000.

Gierens, K., Kohlhepp, R., Spichtinger, P., and SchroedterHomscheidt, M.: Ice supersaturation as seen from TOVS, Atmos. Chem. Phys., 4, 539-547, doi:10.5194/acp-4-539-2004, 2004.

Gierens, K., Spichtinger, P., and Schumann, U.: Ice supersaturation, in: Atmospheric Physics. Background - Methods - Trends, edited by: Schumann, U., chap. 9, 135-150, Springer, Heidelberg, Germany, 2012.

Immler, F., Treffeisen, R., Engelbart, D., Krüger, K., and Schrems, O.: Cirrus, contrails, and ice supersaturated regions in high pressure systems at northern mid latitudes, Atmos. Chem. Phys., 8, 1689-1699, doi:10.5194/acp-8-1689-2008, 2008.

Irvine, E., Hoskins, B., and Shine, K.: The dependence of contrail formation on the weather pattern and altitude in the North Atlantic, Geophys. Res. Lett., 39, L12802, doi:10.1029/2012GL051909, 2012.

Kästner, M., Meyer, R., and Wendling, P.: Influence of weather conditions on the distribution of persistent contrails, Meteorol. Appl., 6, 261-271, 1999.

Lamquin, N., Stubenrauch, C. J., Gierens, K., Burkhardt, U., and Smit, H.: A global climatology of upper-tropospheric ice supersaturation occurrence inferred from the Atmospheric Infrared Sounder calibrated by MOZAIC, Atmos. Chem. Phys., 12, 381405, doi:10.5194/acp-12-381-2012, 2012.

Luo, Z., Kley, D., Johnson, R., and Smit, H.: Ten years of measurements of tropical upper-tropospheric water vapor by MOZAIC. Part I: Climatology, variability, transport, and relation to deep convection, J. Climate, 20, 418-435, 2007.

Luo, Z., Kley, D., Johnson, R., and Smit, H.: Ten years of measurements of tropical upper-tropospheric water vapor by MOZAIC. Part II: Assessing the ECMWF humidity analysis, J. Climate, 21, 1449-1466, 2008. 
Nawrath, S.: Water Vapor in the Tropical Upper Troposphere: On the Influence of Deep Convection, Ph.D. thesis, Universität zu Köln, 2002.

Spichtinger, P.: Eisübersättigte Regionen, Ph.D. thesis, Deutsches Zentrum für Luft- und Raumfahrt, Institut für Physik der Atmosphäre, 2004.

Spichtinger, P., Gierens, K., and Read, W.: The statistical distribution law of relative humidity in the global tropopause region, Meteorol. Z., 11, 83-88, 2002.

Spichtinger, P., Gierens, K., Leiterer, U., and Dier, H.: Ice supersaturation in the tropopause region over Lindenberg, Germany, Meteorol. Z., 12, 143-156, 2003a.

Spichtinger, P., Gierens, K., and Read, W.: The global distribution of ice-supersaturated regions as seen by the Microwave Limb Sounder, Q. J. Roy. Meteor. Soc., 129, 3391-3410, 2003b.

Spichtinger, P., Gierens, K., and Dörnbrack, A.: Formation of ice supersaturation by mesoscale gravity waves, Atmos. Chem. Phys., 5, 1243-1255, doi:10.5194/acp-5-1243-2005, 2005a.
Spichtinger, P., Gierens, K., and Wernli, H.: A case study on the formation and evolution of ice supersaturation in the vicinity of a warm conveyor belt's outflow region, Atmos. Chem. Phys., 5, 973-987, doi:10.5194/acp-5-973-2005, 2005b.

Tompkins, A., Gierens, K., and Rädel, G.: Ice supersaturation in the ECMWF Integrated Forecast System, Q. J. Roy. Meteor. Soc., 133, 53-63, 2007.

Waugh, D.: Impact of potential vorticity intrusions on subtropical upper tropospheric humidity, J. Geophys. Res., 110, D11305, doi:10.1029/2004JD005664, 2005.

Wylie, D.: Cirrus and Weather - A Satellite Perspective, in: Cirrus, edited by: Lynch, D., Sassen, K., Starr, D., and Stephens, G., chap. 6, 136-146, Oxford University Press, 2002. 